\title{
Growth of massive black holes by super-Eddington accretion
}

\author{
T. Kawaguchi ${ }^{1,2, \star}$, K. Aoki ${ }^{3}$, K. Ohta $^{4}$, and S. Collin ${ }^{1}$ \\ ${ }^{1}$ LUTh/Observatoire de Paris-Meudon, 5 Place J. Janssen, 92195 Meudon, France \\ 2 Postdoctoral Fellow of the Japan Society for the Promotion of Science \\ 3 Subaru Telescope, National Astronomical Observatory of Japan, 650 North A'ohoku Place, Hilo, HI 96729, USA \\ ${ }^{4}$ Department of Astronomy, Graduate School of Science, Kyoto University, Sakyo-ku, Kyoto 606-8502, Japan
}

Received 17 March 2004 / Accepted 30 April 2004

\begin{abstract}
Narrow-Line Seyfert 1 galaxies (NLS1s) and Narrow-Line quasars (NLQs) seem to amount to $10-30 \%$ of active galactic nuclei (AGNs) in the local universe. Together with their average accretion rate, we argue that a black hole (BH) growth by factor of 8-800 happens in these super-Eddington accretion phase of AGNs. Moreover, there is a possible, systematic underestimation of accretion rates (in the Eddington unit) due to an overestimation of $\mathrm{BH}$ mass by massive accretion discs for super-Eddington objects. If it is true, the factor of $\mathrm{BH}$ growth above may be larger by order(s) of magnitude. In contrast, the growth factor expected in sub-Eddington phase is only $\sim 2$. Therefore, the cosmic $\mathrm{BH}$ growth by accretion is likely dominated by super-Eddington phase, rather than sub-Eddington phase which is the majority among AGNs.

This analysis is based on the fraction and the average accretion rate of NLS1s and NLQs obtained for $z \lesssim 0.5$. If those numbers are larger at higher redshift (where BHs were probably less grown), super-Eddington accretion would be even more important in the context of cosmic $\mathrm{BH}$ growth history.
\end{abstract}

Key words. accretion, accretion disks - black hole physics - galaxies: active - galaxies: evolution - galaxies: nuclei galaxies: Seyfert

\section{Introduction}

Almost all galaxies in the local universe have supermassive black holes (BHs) in their nuclei, not only active galactic nuclei (AGNs) but also apparently normal galaxies (Kormendy \& Richstone 1995; Magorrian et al. 1998). Luminous quasars with massive BHs are observed till redshift $z \approx 6$, when the universe was less than $10 \%$ of its recent age (e.g., Fan et al. 2003; Vestergaard 2004). However, it is not clear at all when those $\mathrm{BHs}$ have been formed and how their $\mathrm{BH}$ mass $\left(M_{\mathrm{BH}}\right)$ have evolved.

Most of unobscured AGNs show broad Balmer lines of hydrogen ( $F W H M$ of $\mathrm{H} \beta>2000 \mathrm{~km} \mathrm{~s}^{-1}$ ), and they are named as broad line Seyfert 1 galaxies (BLS1s) and quasars. From several independent arguments, the duration of an AGN is thought to be $\sim 10^{8}$ yr (Martini 2004 for a review). And the average accretion rate $\dot{M}$ of BLS1s/quasars is about $3 L_{\mathrm{Edd}} / c^{2}$ (e.g., Fig. 11 of Kawaguchi 2003), where $L_{\text {Edd }}$ is the Eddington luminosity $\left(\sim 10^{46}\left(M_{\mathrm{BH}} / 10^{8} M_{\odot}\right) \mathrm{erg} \mathrm{s}^{-1}\right)$. Thus, BHs increase their mass during $\sim 10^{8}$ year only by a factor of $\sim 2$.

Then, what else can be responsible for the cosmic BH growth? Semi-analytical approaches (e.g., Kauffmann \& Haehnelt 2000) employ a scheme where a BH mass is increased not only by accretion but also by merging of two massive $\mathrm{BHs}$

Send offprint requests to: T. Kawaguchi, e-mail: kawaguti@optik.mtk.nao.ac.jp

* Present Address: National Astronomical Observatory of Japan, Tokyo 181-8588, Japan. during a merger event of galaxies, where the amount of $\mathrm{BH}$ masses is conserved. However, the $\mathrm{BH}$ growth via merging is only expected at high mass BHs (associated in most massive dark halos; Cattaneo 2002), and thus this mechanism is not feasible for BH growth from a seed BH to a Seyfert-level BH ( $\left.\sim 10^{6-8} M_{\odot}\right)$. Moreover, discussions comparing the evolution of luminosity functions (LFs) with the volume mass density of BHs (Sołtan 1982; Chokshi \& Turner 1992) infer that the BH growth by accretion process is enough to explain the local $\mathrm{BH}$ mass density (Fabian \& Iwasawa 1999; Yu \& Tremaine 2002; Elvis et al. 2002). Namely, merging of BHs might be negligible for the cosmic BH growth (Marconi et al. 2004).

There is growing evidence that a certain class of AGNs has a higher Eddington ratio (ratio of bolometric to Eddington luminosity) among the AGN population; Narrow Line Seyfert 1 galaxies (NLS1s) and their high luminosity analogue, NarrowLine QSOs (NLQs). They exhibit relatively narrow Balmer lines only slightly broader than the forbidden lines ( $F W H M$ of $\mathrm{H} \beta<2000 \mathrm{~km} \mathrm{~s}^{-1}$ ), optical Fe II multiplet emission, and low [OIII] $-\mathrm{H}_{\beta}$ flux ratio, <3 (see Pogge 2000). Strong soft X-ray excess (e.g., Pounds et al. 1995; Boller et al. 1996; Boller et al. 2003), and rapid X-ray variability (e.g., Otani et al. 1996; Leighly 1999; Boller et al. 2002; Gallo et al. 2004) are often observed. Those characteristics indicate that they have relatively small $M_{\mathrm{BH}}$ and larger $\dot{M} /\left(L_{\mathrm{Edd}} / c^{2}\right)$ (e.g., Brandt \& Boller 1998; Hayashida 2000; Mineshige et al. 2000). 
At super-Eddington accretion regime, the radiative efficiency per unit mass accretion is expected to decrease due to the onset of photon trapping (Begelman 1978). As a result, the emergent luminosity from an accretion flow starts to saturate at around a few times $L_{\text {Edd }}$ (Abramowicz et al. 1988). Thus, rapid BH growth is possible in super-Eddington sources, NLS1s/NLQs, (Rees 1992; Mineshige et al. 2000; Collin et al. 2002; Kawaguchi 2003). However, the amount of BH growth via super-Eddington accretion is quite ambiguous, since it strongly depends on the assumed duration of NLS1s/NLQs. The terms, "super-Eddington" and "sub-", are used in this Letter when an accretion rate of an AGN is larger and smaller than $\sim 10 L_{\mathrm{Edd}} / c^{2}$, where the emergent bolometric luminosity is about $(0.5-1) L_{\text {Edd }}$.

In this Letter, we assume that the ratio of the durations of a NLS1/NLQ and a BLS1/quasar may be proportional to the relative number ratio of these two classes. Then, a simple algebra taking into account the fraction and average accretion rate of NLS1s/NLQs is discussed, in order to evaluate qualitatively to what extent BHs can grow in super-Eddington accreting AGNs (Sect. 2). It is followed by several discussions in the final section.

\section{Super-Eddington accretion and BH growth}

\subsection{Fraction and duration of NLS1 phase}

A fraction of AGNs are NLS1s and NLQs: $\sim 11 \%$ objects among a heterogeneous sample (Marziani et al. 2003), 15\% among the Early Data Release of the Sloan Digital Sky Survey (SDSS; Williams et al. 2002), and $\sim(31-46) \%$ among soft X-ray selected AGNs (Grupe et al. 1999; Grupe 2004; Salvato et al. 2004). The fraction can be even higher in Extreme UV band; 8 NLS1s out of 14 radio-quiet AGNs (Edelson et al. 1999). This wavelength dependency is likely due to the difference of the spectral energy distribution between NLS1s/NLQs and BLS1s/quasars (Boller et al. 1996; Wang et al. 1996; Laor et al. 1997; see Fig. 14 of Kawaguchi 2003). Most of those NLS1s have a redshift $z$ smaller than 0.5. Here, we employ two numbers, $10 \%$ and $30 \%$, as the relative fraction of the NLS1/NLQ among AGNs.

In general, the duration (or the sum of multiple episodic phases) of an AGN is thought to be $\sim 10^{8} \mathrm{yr}$ from several independent arguments (e.g., Martini 2004; Yu \& Tremaine 2002; Marconi et al. 2004). The duration of each episodic phase must be longer than $10^{4} \mathrm{yr}$, to explain the proximity effect (e.g., Bajtlik et al. 1988) and the sizes of ionization-bounded narrowline regions (Bennert et al. 2002).

If statistical properties of AGNs (e.g., relative number ratio of super-Eddington to sub-Eddington sources) are quasi-steady, the fraction of NLS1s inferred above will be proportional to the duration of NLS1/NLQ phase. In fact, the evolution of AGNs is not so rapid in this redshift range, $z=0.5 \rightarrow 0$, taking $5 \mathrm{Gyr}$ : the number density decreases by a factor of 3 , and the knee of LFs stays at almost the same luminosity (e.g., Boyle et al. 2000; Miyaji et al. 2001; Ueda et al. 2003).

Then, let us simply assume that each NLS1 (or NLQ) has a duration of $\sim 10^{7} \mathrm{yr}$ or $\sim 3 \times 10^{7} \mathrm{yr}$. This number $\left(\sim 10^{7} \mathrm{yr}\right)$ was briefly discussed together with the fraction of NLS1s/NLQs also in a thesis (Grupe 1996). A characteristic timescale for BH growth can be roughly evaluated by (Salpeter 1964)

$$
M_{\mathrm{BH}} / \dot{M}=4.5 \times 10^{8}\left(\frac{\dot{M}}{L_{\mathrm{Edd}} / c^{2}}\right)^{-1} \text { year. }
$$

If $\mathrm{BH}$ growth mainly occures in NLS1/NLQ phase, the timescale of the order of $10^{7} \mathrm{yr}$ implies that $\dot{M} \gtrsim 45 L_{\mathrm{Edd}} / c^{2}$.

\subsection{Accretion rate of NLS1s}

The BH mass and gas accretion rate of AGNs onto their central BHs can be estimated using the observed relation between optical luminosity and the size of broad line region (Wandel et al. 1999; Kaspi et al. 2000). Comparing optical luminosity of NLS1s and NLQs between observation and theoretical models, along with $\mathrm{BH}$ masses inferred from $\mathrm{H} \beta$ and [O III] widths, their accretion rate is about $100 L_{\text {Edd }} / c^{2}$, on average (Fig. 11 in Kawaguchi 2003; Collin et al. 2002).

Alternative and independent method to evaluate $M_{\mathrm{BH}}$ of NLS1s/NLQs is spectral fitting of slim disk models at soft $X$-ray range (Wang \& Netzer 2003). Moreover, if one tries to fit the broad-band spectra at optical-X-ray band, not only $M_{\mathrm{BH}}$ but also $\dot{M}$ are estimated simultaneously (Kawaguchi 2003; Kawaguchi et al. 2004; Kawaguchi et al. in prep.; see Kawaguchi 2004). Currently, these models are the only ones that explain broadband SEDs of NLS1s, in our best knowledge. This kind of estimations indicate $M_{\mathrm{BH}}$ and $\dot{M}$ which are similar to those derived by the line widths in the previous paragraph. Spectral fitting is relatively time consuming, but this success implies that we can use the line widths for quick estimations, as the first order.

\subsection{Linear growth of $\mathrm{BHs}$}

Now, we start with an assumption that the gas accretion rate onto a central BH is almost constant: $\dot{M} \approx$ const. For instance, a following scenario is possible for the constant $-\dot{M}$ case: large amount of gas accretion (super-Eddington accretion phase = NLS1/NLQ-phase) may start induced by an unknown trigger. With a growing $\mathrm{BH}$ mass, the accretion rate in unit of the Eddington rate will be reduced down to sub-Eddington phase (broad line Seyferts and quasars). Accretion rate (in Eddington unit) will decrease continuously, and eventually it will become lower than $\sim 0.2 L_{\text {Edd }} / c^{2}$, followed by a transition from a radiatively efficient to an inefficient flow (i.e. optically-thin advective flow; e.g., Meyer et al. 2000), such as Low Luminosity AGNs.

Employing this view, the growth of $M_{\mathrm{BH}}$ with time $t$ is linear as is expressed below:

$M_{\mathrm{BH}}(t)=M_{\mathrm{BH}}(t=0)+\dot{M} \times t$. 
Substituting the average accretion rate of NLS1s/NLQs to $\dot{M}$, we have

$M_{\mathrm{BH}}\left(t=10^{7} \mathrm{yr}\right) \approx 8 \times M_{\mathrm{BH}}(t=0)$, and

$M_{\mathrm{BH}}\left(t=3 \times 10^{7} \mathrm{yr}\right) \approx 23 \times M_{\mathrm{BH}}(t=0)$,

if $\dot{M}=100 \frac{L_{\mathrm{Edd}}\left(t=10^{6.5} \mathrm{yr}\right)}{c^{2}}$.

On the other hand, in a sub-Eddington phase the factor of $\mathrm{BH}$ growth during $\sim 10^{8} \mathrm{yr}$ is not so significant:

$M_{\mathrm{BH}}\left(t=10^{8} \mathrm{yr}\right)=1.8 \times M_{\mathrm{BH}}(t=0)$,

if $\dot{M}=3 \frac{L_{\mathrm{Edd}}\left(t=10^{7.5} \mathrm{yr}\right)}{c^{2}}$.

\subsection{Exponential growth of $\mathrm{BHs}$}

Next, another assumption on accretion rate is that $\dot{M} /\left(L_{\text {Edd }} / c^{2}\right)$ may be constant with time by some unknown self-regulation mechanism(s). In this case, sub-Eddington phase does not follow at the end of super-Eddington phase, contrary to the previous case. Instead, in each trigger of AGN activity, $\dot{M} /\left(L_{\mathrm{Edd}} / c^{2}\right)$ is (somehow randomly) chosen: if a nuclei has a superEddington (sub-) accretion rate it will appear as a NLS1/NLQ (a broad-line Seyfert/quasar). The growth of $M_{\mathrm{BH}}$ will be exponential with this assumption, and is written as,

$$
\begin{aligned}
M_{\mathrm{BH}}(t) & =M_{\mathrm{BH}}(t=0) \times \exp \left[t \times\left(\frac{\dot{M}}{M_{\mathrm{BH}}}\right)\right] \\
& =M_{\mathrm{BH}}(t=0) \times \exp \left[\left(\frac{t}{4.5 \times 10^{8} \mathrm{yr}}\right)\left(\frac{\dot{M}}{L_{\mathrm{Edd}} / c^{2}}\right)\right] .
\end{aligned}
$$

Substituting the average accretion rate (Sect. 2.2), we get

$$
\begin{aligned}
& M_{\mathrm{BH}}\left(t=10^{7} \mathrm{yr}\right) \approx 9 \times M_{\mathrm{BH}}(t=0), \text { and } \\
& M_{\mathrm{BH}}\left(t=3 \times 10^{7} \mathrm{yr}\right) \approx 800 \times M_{\mathrm{BH}}(t=0) .
\end{aligned}
$$

Given the average accretion rate $\left(\sim 3 L_{\mathrm{Edd}} / c^{2}\right)$ and duration $\left(\sim 10^{8} \mathrm{yr}\right)$ of BLS1s/quasars, a BH can grow by a factor of 1.9 in the sub-Eddington phase.

\section{Discussions and conclusions}

In principle, a $\mathrm{BH}$ mass based on line widths is the mass inside the broad line region, and does not necessarily equal to the true $M_{\mathrm{BH}}$. Outer part of accretion disks in NLS1s/NLQs, where optical continuum is emitted, are self-gravitating (Kawaguchi et al. 2004). Such disks are quite massive, and $M_{\mathrm{BH}}$ based on line widths can be systematically overestimated by a factor of $\sim 2$. If this is taken account into $M_{\mathrm{BH}}$ and $\dot{M}$ estimations, the average accretion rate of NLS1s/NLQs might be $200 L_{\mathrm{Edd}} / c^{2}$, rather than $100 L_{\mathrm{Edd}} / c^{2}$. Thus, the factor of $\mathrm{BH}$ growth presented in Sect. 2.4 (Eqs. (6) and (7)) will be multiplied by themselves. While, it is too efficient to grow a $\mathrm{BH}$ with this accretion rate for a constant- $\dot{M}$ case (Sect. 2.3). For sub-Eddington systems, on the other hand, there is no constraints upon the presence of outer, massive, self-gravitating disks, since such region (if exists) radiates at Near-IR where the emission from dusty torus dominates.

We have utilized the relative fraction and the average accretion rate of NLS1s/NLQs obtained for $z \lesssim 0.5$. Apparently, the Eddington ratios of AGNs detected in SDSS tend to increase with redshift (McLure \& Dunlop 2003). Thus, BH growth via super-Eddington accretion would be much more important in the young universe than discussed above.

The tight correlation between spheroid mass of galaxies and $\mathrm{BH}$ mass indicates that they co-evolve. Indeed, high star formation activities in host galaxies of NLS1s/NLQs are inferred (e.g., Moran et al. 1996; Kawaguchi \& Aoki 2001). Since the gas accretion rate of NLS1s/NLQs is $\sim 2 M_{\odot} \mathrm{yr}^{-1}$ at most (Collin \& Kawaguchi 2004), a small fraction of mass loss by starburst streaming toward the central $\mathrm{BH}$ is enough to form a NLS1/NLQ.

We note that the super-Eddington accretion phase (with $L \lesssim L_{\text {Edd }}$ ) discussed here is not a surprising assumption in the context of cosmic structure formation history. In semianalytical approaches (e.g., Kauffmann \& Haehnelt 2000), for instance, most of accretion events happen in super-Eddington regime if the episodic phase of an AGN is assumed to be less than $10^{8}$ yr (Cattaneo 2001).

For quasars, with their bolometric luminosity $L \gtrsim$ $10^{46} \mathrm{erg} / \mathrm{s}$, LFs can be obtained by direct observations to high redshifts in a wide range of wavebands. Comparing the integration of those LFs with the volume mass density of BHs in the local universe, the radiative efficiency seems to be quite high $(\gtrsim 0.15)$ in those luminous objects (e.g., Yu \& Tremaine 2002; Elvis et al. 2002). Such a high efficiency indicates that most BHs of quasars are rotating with sub-Eddington accretion rates. However, the mass density of local BHs are dominated by quite massive BHs at the knee of mass functions or LFs. Therefore, constraints upon $\mathrm{BH}$ growth based on above discussions are likely valid at high mass end; e.g., $\mathrm{BH}$ growth from $10^{8} M_{\odot}$ to $10^{10} M_{\odot}$.

On the other hand, $\mathrm{BH}$ growth for lower luminous objects with $M_{\mathrm{BH}} \lesssim 10^{8} \quad M_{\odot}$ is quite unclear. The way BHs grow in Seyferts is not necesarilly the same as that of quasars. Actually, Eddington ratios of AGNs seems to be higher for objects with smaller $M_{\mathrm{BH}}$ (e.g., Collin et al. 2002; Collin \& Kawaguchi 2004). Yu \& Tremaine (2002) argued that relatively low luminous AGNs should have less radiative efficiency, $\lesssim 0.1$. Thus, super-Eddington accretion (which is expected to have very low efficiency) seems to play an important role in $\mathrm{BH}$ growth from seed BHs to $\sim 10^{8} M_{\odot}$. This is not in conflict with the idea above that $\mathrm{BHs}$ in quasars grow with high radiative efficiency (via sub-Eddington accretion).

Given that a $\mathrm{BH}$ mass increases by the factors mentioned above, is it enough to grow BHs found in quasars with $\sim 10^{8-10} M_{\odot}$ from seed BHs? It may be possible to make such supermassive BHs if i) the mass of seed BHs at the center of galaxies is large enough (e.g., $\gtrsim 10^{6} M_{\odot}$ ), and/or ii) the duration and average accretion rate of super-Eddinton accreting AGNs are much larger at high redshift than those estimated for local objects. Instead, iii) $\mathrm{BH}$ growth at the highest mass end may be indeed dominated by merging events as expected in semianalytical approaches (Cattaneo 2002). 
In summary, we have argued that the growth of black hole (BH) mass by factor of 8-800 happens in the super-Eddington accretion phase. There is a possible, systematic underestimation of $\dot{M} /\left(L_{\mathrm{Edd}} / c^{2}\right)$ by a factor of $\sim 2$ due to an overestimation of BH mass by massive accretion discs for super-Eddington objects. If it is true, the factor of $\mathrm{BH}$ growth above may be larger by order(s) of magnitude. On the other hand, the growth factor expected in sub-Eddington active galactic nuclei (AGNs) is only $\sim 2$. Therefore, the cosmic BH growth by accretion is dominated by super-Eddington phase, rather than sub-Eddington phase which is the majority among AGNs. These values are based on the fraction and the average accretion rate of NarrowLine Seyfert 1 galaxies and Narrow-Line quasars obtained for $z \lesssim 0.5$. If those numbers are larger at higher redshift (where $\mathrm{BHs}$ are less grown), $\mathrm{BH}$ growth via super-Eddington accretion would be even more important.

For further analysis, we need i) volume limited samples of AGNs to derive an accurate relative fraction of NLS1s/NLQs, and ii) constraints on $z$-dependency of the relative fraction and the average $\dot{M} /\left(L_{\mathrm{Edd}} / c^{2}\right)$ to determine the importance of superEddington accretion in $\mathrm{BH}$ growth history. In this Letter, we divide radio-quiet type I AGNs into two subclasses: objects with $\dot{M} \gtrsim$ or $\lesssim 10 L_{\mathrm{Edd}} / c^{2}$. It would be more appropriate to divide into fine bins for dealing with the observed continuous distribution of $\dot{M} /\left(L_{\mathrm{Edd}} / c^{2}\right)$; e.g., bins for $10-100 L_{\mathrm{Edd}} / c^{2}$ and $100-1000 L_{\mathrm{Edd}} / c^{2}$, etc. For this aim, iii) more sophysticated (with less uncertainty) methods for $\dot{M} /\left(L_{\text {Edd }} / c^{2}\right)$ estimations are necesary.

Acknowledgements. We thank Masayuki Umemura, Nozomu Kawakatu and Andrea Cattaneo for illuminating discussions. Useful comments by the referee, Thomas Boller, are also appreciated. T.K. is supported by the Japan Society for the Promotion of Science (JSPS) Postdoctoral Fellowships for Research Abroad (464).

\section{References}

Abramowicz, M. A., Czerny, B., Lasota, J. P., \& Szuszkiewicz, E. 1988, ApJ, 332, 646

Bajtlik, S., Duncan, R. C., \& Ostriker, J. P. 1988, ApJ, 327, 570

Begelman, M. C. 1978, MNRAS, 184, 53

Bennert, N., Falcke, H., Schulz, H., et al. 2002, ApJ, 574, L105

Boller, Th., Brandt, W. N., Fink, H. H. 1996, A\&A, 305, 53

Boller, Th., Fabian, A. C., Sunyaev, R., et al. 2002, MNRAS, 329, L1

Boller, Th., Tanaka, Y., Fabian, A. C., et al. 2003, MNRAS, 343, L89

Boyle, B. J., Shanks, T., Croom, S. M., et al. 2000, MNRAS, 317, 1014

Brandt, N., \& Boller, T. 1998, Astron. Nach., 319, 163

Cattaneo, A. 2001, MNRAS, 324, 128

Cattaneo, A. 2002, MNRAS, 333, 353

Chokshi, A., \& Turner E. L. 1992, MNRAS, 259, 421

Collin, S., Boisson, C., Mouchet, M., et al. 2002, A\&A, 388, 771

Collin, S., \& Kawaguchi, T. 2004, A\&A, submitted

Edelson, R., Vaughan, S., Warwick, R., Puchnarewicz, E., \& George, I. 1999, MNRAS, 307, 91
Elvis, M., Risalti, G., \& Zamorani, G. 2002, ApJL, 565, 75

Fabian, A., \& Iwasawa, K. 1999, MNRAS, 303, 34

Fan, X., Strauss, M. A. \& Schneider, D. P. 2003, AJ, 125, 1649

Gallo, L. C., Boller, Th., Tanaka, Y., et al. 2004, MNRAS, 347, 269

Grupe, D. 1996, Ph.D. Thesis, Göttingen University

Grupe, D., Beuermann, K., Mannheim, K., \& Thomas, H.-C. 1999, A\&A, 350, 805

Grupe, D. 2004, AJ, in press [arXiv: astro-ph/0401167]

Hayashida, K. 2000, New Astron. Rev., 44, 419

Kaspi, S., Smith, P. S., Netzer, H., et al. 2000, ApJ, 533, 631

Kauffmann, G., \& Haehnelt, M. G. 2000, MNRAS, 311, 576

Kawaguchi, T. 2003, ApJ, 593, 69

Kawaguchi, T. 2004, in Stellar-Mass, Intermediate -Mass, and Supermassive Black Holes, ed. K. Makishima \& S. Mineshige, Progress of Theoretical Physics Supplement, in press

Kawaguchi, T., \& Aoki, K. 2001, in Advanced Lectures on the Starburst-AGN Connection, ed. R. Mujica, I. Aretxaga, \& A. D. Kunth (Electronic Edition), 51; available at http://www . inaoep.mx/agn $00 /$ posters/kawaguchi.ps.gz

Kawaguchi, T., Pierens, A., \& Huré, J.-M. 2004, A\&A, 415, 47

Kormendy, J., \& Richstone, D. 1995, ARA\&A, 33, 581

Laor, A., Fiore F., Elvis M., et al. 1997, ApJ, 477, 93

Leighly, K. M. 1999, ApJS, 125, 297

Magorrian, J., Tremaine, S., \& Richstone, D. 1998, AJ, 115, 2285

Marconi, A., Risaliti G., Gilli, R., et al. 2004, MNRAS in press [arXiv: astro-ph/0311619]

Martini, P. 2004, in Carnegie Observatories Astrophysics Series, Vol. 1: Coevolution of Black Holes and Galaxies, ed. L. C. Ho (Cambridge: Cambridge Univ. Press), in press [arXiv:astro-ph/0304009]

Marziani, P., Sulentic, J. W., Zamanov, R., et al. 2003, ApJS, 145, 199

McLure, R. J. \& Dunlop, J. S. 2003, MNRAS, submitted [arXiv:astro-ph/0310267]

Meyer, F., Liu, B. F., \& Meyer-Hofmeister, E. 2000, A\&A, 354, L67

Mineshige, S., Kawaguchi, T., Takeuchi, M., \& Hayashida, K. 2000, PASJ, 52, 499

Miyaji, T., Hasinger, G., \& Schmidt, M. 2001, A\&A, 369, 49

Moran, E. C., Halpern, J. P., \& Helfand, D. J. 1996, ApJS, 106, 341

Otani, C., Kii, T., Miya, K. 1996 in Röntgenstrahlung from the Universe (MPE Report 263), ed. H. U. Zimmermann, J. E. Trümper, H. Yorke (Garching, MPE Press), 491

Pogge, R. W. 2000, New Astron. Rev., 44, 381

Pounds, K. A., Done, C., Osborne, J. 1995, MNRAS 277, L5

Rees, M. J. 1992, in Physics of Active Galactic Nuclei, ed. W.J. Duschl, S.J. Wagner (Berlin, Springer-Verlag), 662

Salpeter, E. E. 1964, ApJ, 140, 796

Salvato, M., Greiner, J., \& Kuhlbrodt, B. 2004, ApJL, 600, 31

Sołtan, A. 1982, MNRAS, 200, 115

Ueda, Y. Akiyama, M., Ohta, K., \& Miyaji, T. 2003, ApJ, 598, 886

Yu, Q., \& Tremaine, S. 2002, MNRAS, 335, 965

Vestergaard, M. 2004, ApJ, 601, 676

Wandel, A., Peterson, B. M., \& Malkan, M. A. 1999, ApJ, 526, 579

Wang, J.-M., \& Netzer, H. 2003, A\&A, 398, 927

Wang, T., Brinkmann, W., \& Bergeron J. 1996, A\&A, 309, 81

Williams, R. J., Pogge, R. W., \& Mathur, S. 2002, AJ, 124, 3042 